DESY 10-059

\title{
Impact of squark generation mixing on the search for squarks decaying into fermions at LHC
}

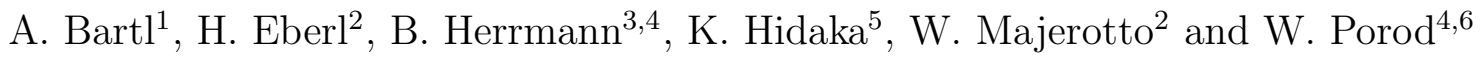 \\ 1 Fakultät für Physik, Universität Wien, A-1090 Vienna, Austria \\ 2 Institut für Hochenergiephysik der Österreichischen Akademie der Wissenschaften, \\ A-1050 Vienna, Austria \\ 3 Deutsches Elektronen-Synchrotron (DESY), Theory Group, D-22603 Hamburg, \\ Germany \\ 4 Institut für Theoretische Physik und Astrophysik, Universität Würzburg, D-97074 \\ Würzburg, Germany \\ ${ }^{5}$ Department of Physics, Tokyo Gakugei University, Koganei, Tokyo 184-8501, Japan \\ 6 AHEP Group, Instituto de Fisica Corpuscular - C.S.I.C., Universidad de València, \\ E-46071 València, Spain
}

\begin{abstract}
We study the effect of squark generation mixing on squark production and decays at LHC in the Minimal Supersymmetric Standard Model (MSSM). We show that the effect can be very large despite the very strong constraints on quark-flavour violation $(\mathrm{QFV})$ from experimental data on $\mathrm{B}$ mesons. We find that the two lightest up-type squarks $\tilde{u}_{1,2}$ can have large branching ratios for the decays into $c \tilde{\chi}_{1}^{0}$ and $t \tilde{\chi}_{1}^{0}$ at the same time due to squark generation mixing, leading to QFV signals ' $p p \rightarrow c \bar{t}(t \bar{c})+$ missing- $E_{T}+X$ ' with a significant rate. The observation of this remarkable signature would provide a powerful test of supersymmetric QFV at LHC. This could have a significant impact on the search for squarks and the determination of the underlying MSSM parameters.
\end{abstract}




\section{Introduction}

The exploration of the TeV scale has begun with the start up of the LHC run. Gluinos and squarks, the supersymmetric partners of gluons and quarks, will be produced copiously for masses up to $O(1 \mathrm{TeV})$ if supersymmetry (SUSY) is realized in nature. After the discovery of SUSY, the determination of SUSY parameters will be one of the main experimental programs. The determination of the soft-SUSY-breaking parameters will be particularly important to pin down the SUSY-breaking mechanism. As the softSUSY-breaking terms are the source of flavour violation beyond the Standard Model (SM), the measurement of flavour violating observables is directly linked to the crucial question about the SUSY-breaking mechanism. It is usually assumed that production and decays of gluinos and squarks are quark-flavour conserving (QFC). However, additional flavour structures (i.e. squark generation mixings) would imply that squarks are not quark-flavour eigenstates, which could result in sizable quark-flavour violation (QFV) effects significantly larger than those due to the Cabibbo-Kobayashi-Maskawa (CKM) mixing.

Additional flavour structures will of course give contributions to flavour-changing neutral current (FCNC) processes. Up to now all measurements of such processes are consistent with the SM predictions, which in turn requires that the flavour structure of new physics at the $\mathrm{TeV}$ scale is highly constrained. In particular, this flavour structure could be closely related to the flavour structure of the SM Yukawa couplings. The most extreme case is minimal flavour violation (MFV) $[1,2,3]$ which assumes that the Yukawa coupling matrices of the SM are the only source of flavour violation even in interactions involving new particles. Supersymmetric models of this kind are gauge-mediated SUSY-breaking or minimal supergravity (mSUGRA) models with universal boundary conditions [4]. However, while the flavour constraints suggest that the dominant flavour structure of new physics should be MFV, there is certainly room for sub-dominant contributions that are not MFV. The discovery of such non-MFV (NMFV) physics will be of utmost interest. There are also known examples of flavour models which do have large flavour violating entries in the squark sector getting consistency with the flavour observables in a different way. An example is a model with an extended R-symmetry [5] where the left-right squark mixing terms are absent and the gauginos are Dirac particles. Another possibility would be hybrid gauge and gravity mediation of supersymmetry breaking [6] where one gets sizable NMFV contributions 
(i.e. sizable squark generation mixing terms) as discussed in [7].

The effect of QFV in the squark sector on reactions with external particles being SM particles [8, 9] (or SUSY Higgs bosons [10]) has been studied in several publications. In this case the effect of QFV in the squark sector is induced only by SUSY particle (sparticle) loops.

However, in reactions with external SUSY particles, the QFV effect can already occur at tree-level and hence can be rather large. The QFV decay $\tilde{t}_{1} \rightarrow c \tilde{\chi}_{1}^{0}$ [11] and QFV gluino decays [12] were studied in the scenario of MFV, where the only source of QFV is the mixing due to the CKM matrix. The QFV decay $\tilde{t}_{1} \rightarrow c \tilde{\chi}_{1}^{0}$ is actually the standard search mode at the Tevatron for light top-squarks if their decays into bottom-quark plus chargino and top-quark plus neutralino are kinematically forbidden. Squark pair production and their decays at LHC have been analyzed in scenarios of NMFV, where the effect of the squark generation mixing is also included $[13,14]$. QFV gluino decays [15] and QFV squark decays [16] have been studied in the Minimal Supersymmetric Standard Model (MSSM) with squark generation mixing in its most general form.

In the present paper, we study the effect of QFV due to the mixing of charmsquarks and top-squarks both on production and subsequent decays of squarks in the general MSSM with R parity conservation. In principle also the mixing between right up-squark and left top-squark is hardly constrained as pointed out in [17]. Here for simplicity we do not take into account such a mixing as we are mainly interested in demonstrating the main QFV effects and signals. Note that in case one cannot distinguish between the quarks of the first two generations, the corresponding QFV signals will involve jets whose original quark is not identified, and hence the effects of the two mixings (i.e. the 1st and 3rd generation mixing and the 2 nd and 3rd generation mixing) cannot be distinguished.

We show that the QFV squark decay branching ratios $\mathrm{B}\left(\tilde{u}_{i} \rightarrow c \tilde{\chi}_{1}^{0}\right)$ and $\mathrm{B}\left(\tilde{u}_{i} \rightarrow\right.$ $\left.t \tilde{\chi}_{1}^{0}\right)(i=1,2)$ can be very large (up to $\sim 50 \%$ ) simultaneously due to the squark generation mixing in a significant region of the QFV parameters despite the very strong experimental constraints from B factories, Tevatron and LEP. Here $\tilde{u}_{1,2}$ are the two lightest up-type squarks and $\tilde{\chi}_{1}^{0}$ is the lightest neutralino. This leads to QFV signal events ' $p p \rightarrow c \bar{t}(\bar{c} t)+E_{T}^{m i s}+X^{\prime}$ and ' $p p \rightarrow t t(\bar{t} \bar{t})+E_{T}^{m i s}+X^{\prime}$ at LHC, which we also study in the present article, where $E_{T}^{m i s}$ is the missing transverse energy. 


\section{Squark mixing with flavour violation}

The most general up-type squark mass matrix including left-right mixing as well as quark-flavour mixing in the super-CKM basis of $\tilde{u}_{0 \gamma}=\left(\tilde{u}_{L}, \tilde{c}_{L}, \tilde{t}_{L}, \tilde{u}_{R}, \tilde{c}_{R}, \tilde{t}_{R}\right), \gamma=$ $1, \ldots, 6$, is $[18]$

$$
M_{\tilde{u}}^{2}=\left(\begin{array}{cc}
M_{\tilde{u} L L}^{2} & \left(M_{\tilde{u} R L}^{2}\right)^{\dagger} \\
M_{\tilde{u} R L}^{2} & M_{\tilde{u} R R}^{2}
\end{array}\right),
$$

where the three $3 \times 3$ matrices read

$$
\begin{aligned}
\left(M_{\tilde{u} L L}^{2}\right)_{\alpha \beta} & =M_{Q_{u} \alpha \beta}^{2}+\left[\left(\frac{1}{2}-\frac{2}{3} \sin ^{2} \theta_{W}\right) \cos 2 \beta m_{Z}^{2}+m_{u_{\alpha}}^{2}\right] \delta_{\alpha \beta}, \\
\left(M_{\tilde{u} R R}^{2}\right)_{\alpha \beta} & =M_{U \alpha \beta}^{2}+\left[\frac{2}{3} \sin ^{2} \theta_{W} \cos 2 \beta m_{Z}^{2}+m_{u_{\alpha}}^{2}\right] \delta_{\alpha \beta}, \\
\left(M_{\tilde{u} R L}^{2}\right)_{\alpha \beta} & =\left(v_{2} / \sqrt{2}\right) T_{U \beta \alpha}-m_{u_{\alpha}} \mu^{*} \cot \beta \delta_{\alpha \beta} .
\end{aligned}
$$

The indices $\alpha, \beta=1,2,3$ characterize the quark flavours $u, c, t$, respectively. $M_{Q_{u}}^{2}$ and $M_{U}^{2}$ are the hermitian soft-SUSY-breaking mass matrices for the left and right up-type squarks, respectively. Note that in the super-CKM basis one has $M_{Q_{u}}^{2}=K \cdot M_{Q}^{2} \cdot K^{\dagger}$ due to the $\mathrm{SU}(2)$ symmetry, where $M_{Q}^{2}$ is the hermitian soft-SUSY-breaking mass matrix for the left down-type squarks and $K$ is the CKM matrix. Note also that $M_{Q_{u}}^{2} \simeq M_{Q}^{2}$ as $K \simeq 1 . T_{U}$ is the soft-SUSY-breaking trilinear coupling matrix of the up-type squarks: $\mathcal{L}_{\text {int }}=-\left(T_{U \alpha \beta} \tilde{u}_{R \beta}^{\dagger} \tilde{u}_{L \alpha} H_{2}^{0}+\right.$ h.c. $)+\cdots, \mu$ is the higgsino mass parameter. $v_{1,2}$ are the vacuum expectation values of the Higgs fields with $v_{1,2} / \sqrt{2} \equiv\left\langle H_{1,2}^{0}\right\rangle$, and $\tan \beta \equiv v_{2} / v_{1} . m_{u_{\alpha}}\left(u_{\alpha}=u, c, t\right)$ are the quark masses.

The physical mass eigenstates $\tilde{u}_{i}, i=1, \ldots, 6$, are given by $\tilde{u}_{i}=R_{i \alpha}^{\tilde{u}} \tilde{u}_{0 \alpha}$. The mixing matrix $R^{\tilde{u}}$ and the mass eigenvalues are obtained by a unitary transformation $R^{\tilde{u}} M_{\tilde{u}}^{2} R^{\tilde{u} \dagger}=\operatorname{diag}\left(m_{\tilde{u}_{1}}, \ldots, m_{\tilde{u}_{6}}\right)$, where $m_{\tilde{u}_{i}}<m_{\tilde{u}_{j}}$ for $i<j$.

Having in mind that $M_{Q_{u}}^{2} \simeq M_{Q}^{2}$, we define the QFV parameters $\delta_{\alpha \beta}^{u L L}, \delta_{\alpha \beta}^{u R R}$ and $\delta_{\alpha \beta}^{u R L}$ $(\alpha \neq \beta)$ as follows [19]:

$$
\delta_{\alpha \beta}^{u L L} \equiv M_{Q \alpha \beta}^{2} / \sqrt{M_{Q \alpha \alpha}^{2} M_{Q \beta \beta}^{2}},
$$




$$
\begin{aligned}
\delta_{\alpha \beta}^{u R R} & \equiv M_{U \alpha \beta}^{2} / \sqrt{M_{U \alpha \alpha}^{2} M_{U \beta \beta}^{2}}, \\
\delta_{\alpha \beta}^{u R L} & \equiv\left(v_{2} / \sqrt{2}\right) T_{U \beta \alpha} / \sqrt{M_{U \alpha \alpha}^{2} M_{Q \beta \beta}^{2}} .
\end{aligned}
$$

The relevant QFV parameters in this study are $\delta_{23}^{u L L}, \delta_{23}^{u R R}, \delta_{23}^{u R L}$ and $\delta_{32}^{u R L}$ which are the $\tilde{c}_{L}-\tilde{t}_{L}, \tilde{c}_{R}-\tilde{t}_{R}, \tilde{c}_{R}-\tilde{t}_{L}$ and $\tilde{c}_{L}-\tilde{t}_{R}$ mixing parameters, respectively. The downtype squark mass matrix can be parameterized analogously to the up-type squark mass matrix [18].

The properties of the charginos $\tilde{\chi}_{i}^{ \pm}\left(i=1,2, m_{\tilde{\chi}_{1}^{ \pm}}<m_{\tilde{\chi}_{2}^{ \pm}}\right)$and neutralinos $\tilde{\chi}_{k}^{0}$ $\left(k=1, \ldots, 4, m_{\tilde{\chi}_{1}^{0}}<\ldots<m_{\tilde{\chi}_{4}^{0}}\right)$ are determined by the parameters $M_{2}, M_{1}, \mu$ and $\tan \beta$, where $M_{2}$ and $M_{1}$ are the $\mathrm{SU}(2)$ and $\mathrm{U}(1)$ gaugino mass parameters, respectively.

\section{Constraints}

In our analysis, we impose the following conditions on the MSSM parameter space in order to respect experimental and theoretical constraints:

(i) Constraints from the B-physics experiments relevant mainly for the mixing between the second and third generations of squarks:

$B(b \rightarrow s \gamma)=\left(3.57 \pm\left((0.24 \times 1.96)^{2}+(0.23 \times 1.96)^{2}\right)^{1 / 2}\right) \times 10^{-4}=(3.57 \pm$ $0.65) \times 10^{-4}(95 \% \mathrm{CL})$, where we have combined the experimental error of $0.24 \times 1.96 \times 10^{-4}(95 \% \mathrm{CL})[20]$ quadratically with the theoretical uncertainty of $0.23 \times 1.96 \times 10^{-4}(95 \% \mathrm{CL})[21], 0.60 \times 10^{-6}<B\left(b \rightarrow s l^{+} l^{-}\right)<2.60 \times 10^{-6}$ with $l=e$ or $\mu(95 \% \mathrm{CL})[22], B\left(B_{s} \rightarrow \mu^{+} \mu^{-}\right)<4.3 \times 10^{-8}(95 \% \mathrm{CL})[20], \mid R_{B \tau \nu}^{S U S Y}-$ $1.35 \mid<0.76(95 \% \mathrm{CL})$ with $R_{B \tau \nu}^{S U S Y} \equiv B^{S U S Y}\left(B_{u}^{-} \rightarrow \tau^{-} \bar{\nu}_{\tau}\right) / B^{S M}\left(B_{u}^{-} \rightarrow \tau^{-} \bar{\nu}_{\tau}\right) \simeq$ $\left(1-\left(\frac{m_{B^{+}} \tan \beta}{m_{H^{+}}}\right)^{2}\right)^{2}[23]$. Moreover we impose the following condition on the SUSY prediction: $\left|\Delta M_{B_{s}}^{S U S Y}-17.77\right|<\left((0.12 \times 1.96)^{2}+3.3^{2}\right)^{1 / 2} \mathrm{ps}^{-1}=3.31 \mathrm{ps}^{-1}(95 \%$ $\mathrm{CL})$, where we have combined the experimental error of $0.12 \times 1.96 \mathrm{ps}^{-1}(95 \%$ CL) [24] quadratically with the theoretical uncertainty of $3.3 \mathrm{ps}^{-1}$ (95\% CL) [25].

(ii) The experimental limit on SUSY contributions to the electroweak $\rho$ parameter [26]: $\Delta \rho(S U S Y)<0.0012$.

(iii) The LEP limits on the SUSY particle masses [27]: $m_{\tilde{\chi}_{1}^{ \pm}}>103 \mathrm{GeV}, m_{\tilde{\chi}_{1}^{0}}>50$ 
$\mathrm{GeV}, m_{\tilde{u}_{1}, \tilde{d}_{1}}>100 \mathrm{GeV}, m_{\tilde{u}_{1}, \tilde{d}_{1}}>m_{\tilde{\chi}_{1}^{0}}, m_{A^{0}}>93 \mathrm{GeV}, m_{h^{0}}>110 \mathrm{GeV}$, where $A^{0}$ is the CP-odd Higgs boson and $h^{0}$ is the lighter CP-even Higgs boson.

(iv) The Tevatron limits on the gluino and squark masses [28].

(v) The vacuum stability conditions for the trilinear coupling matrix [29]:

$$
\begin{aligned}
\left|T_{U \alpha \alpha}\right|^{2} & <3 Y_{U \alpha}^{2}\left(M_{Q_{u} \alpha \alpha}^{2}+M_{U \alpha \alpha}^{2}+m_{2}^{2}\right), \\
\left|T_{D \alpha \alpha}\right|^{2} & <3 Y_{D \alpha}^{2}\left(M_{Q \alpha \alpha}^{2}+M_{D \alpha \alpha}^{2}+m_{1}^{2}\right), \\
\left|T_{U \alpha \beta}\right|^{2} & <Y_{U \gamma}^{2}\left(M_{Q_{u} \alpha \alpha}^{2}+M_{U \beta \beta}^{2}+m_{2}^{2}\right), \\
\left|T_{D \alpha \beta}\right|^{2} & <Y_{D \gamma}^{2}\left(M_{Q \alpha \alpha}^{2}+M_{D \beta \beta}^{2}+m_{1}^{2}\right),
\end{aligned}
$$

with $(\alpha \neq \beta$; $\gamma=\operatorname{Max}(\alpha, \beta) ; \alpha, \beta=1,2,3)$ and $m_{1}^{2}=\left(m_{H^{ \pm}}^{2}+m_{Z}^{2} \sin ^{2} \theta_{W}\right) \sin ^{2} \beta-$ $\frac{1}{2} m_{Z}^{2}, m_{2}^{2}=\left(m_{H^{ \pm}}^{2}+m_{Z}^{2} \sin ^{2} \theta_{W}\right) \cos ^{2} \beta-\frac{1}{2} m_{Z}^{2}$. The Yukawa couplings of the uptype and down-type quarks are $Y_{U \alpha}=\sqrt{2} m_{u_{\alpha}} / v_{2}=\frac{g}{\sqrt{2}} \frac{m_{u_{\alpha}}}{m_{W} \sin \beta}\left(u_{\alpha}=u, c, t\right)$ and $Y_{D \alpha}=\sqrt{2} m_{d_{\alpha}} / v_{1}=\frac{g}{\sqrt{2}} \frac{m_{d_{\alpha}}}{m_{W} \cos \beta}\left(d_{\alpha}=d, s, b\right)$, with $m_{u_{\alpha}}$ and $m_{d_{\alpha}}$ being the running quark masses at the weak scale and $g$ the $\mathrm{SU}(2)$ gauge coupling. All soft-SUSY-breaking parameters are assumed to be given at the weak scale. As SM parameters we take $m_{W}=80.4 \mathrm{GeV}, m_{Z}=91.2 \mathrm{GeV}$ and the on-shell top-quark mass $m_{t}=174.3 \mathrm{GeV}$. We have found that our results shown in the following are fairly insensitive to the precise value of $m_{t}$.

We calculate the observables in (i)-(iv) by using the public code SPheno v3.0 [30]. Condition (i) except for $B\left(B_{u}^{-} \rightarrow \tau^{-} \bar{\nu}_{\tau}\right)$ strongly constrains the 2nd and 3rd generation squark mixing parameters $M_{Q 23}^{2}, M_{U 23}^{2}, M_{D 23}^{2}, T_{U 23}, T_{D 23}$ and $T_{D 32}$. The constraints from $B(b \rightarrow s \gamma)$ and $\Delta M_{B_{s}}$ are especially important [16]. $B(b \rightarrow s \gamma)$ is sensitive to $M_{Q 23}^{2}, T_{U 23}, T_{D 23}$ and $\Delta M_{B_{s}}$ is sensitive to $M_{Q 23}^{2} \cdot M_{U 23}^{2}, M_{Q 23}^{2} \cdot M_{D 23}^{2}$. 


\begin{tabular}{|c||c|c|c|c|c|c|c|c|c|}
\hline \multicolumn{1}{|c||}{$M_{Q \alpha \beta}^{2}$} & $\beta=1$ & $\beta=2$ & $\beta=3$ \\
\hline \hline$\alpha=1$ & $(920)^{2}$ & 0 & 0 \\
\hline$\alpha=2$ & 0 & $(880)^{2}$ & $(224)^{2}$ \\
\hline$\alpha=3$ & 0 & $(224)^{2}$ & $(840)^{2}$ \\
\hline \hline 139 & $M_{1}$ & $M_{2}$ & $M_{3}$ & $\mu$ & $\tan \beta$ & $m_{A^{0}}$ \\
\hline & & 800 & 1000 & 10 & 800 \\
\hline
\end{tabular}

\begin{tabular}{|c||c|c|c|}
\hline$M_{D \alpha \beta}^{2}$ & $\beta=1$ & $\beta=2$ & $\beta=3$ \\
\hline \hline$\alpha=1$ & $(830)^{2}$ & 0 & 0 \\
\hline$\alpha=2$ & 0 & $(820)^{2}$ & 0 \\
\hline$\alpha=3$ & 0 & 0 & $(810)^{2}$ \\
\hline
\end{tabular}

\begin{tabular}{|c||c|c|c|}
\hline$M_{U \alpha \beta}^{2}$ & $\beta=1$ & $\beta=2$ & $\beta=3$ \\
\hline \hline$\alpha=1$ & $(820)^{2}$ & 0 & 0 \\
\hline$\alpha=2$ & 0 & $(600)^{2}$ & $(373)^{2}$ \\
\hline$\alpha=3$ & 0 & $(373)^{2}$ & $(580)^{2}$ \\
\hline
\end{tabular}

Table 1: The basic MSSM parameters in our reference scenario with QFV. All of $T_{U \alpha \beta}$ and $T_{D \alpha \beta}$ are set to zero. All mass parameters are given in $\mathrm{GeV}$.

\section{Flavour violating fermionic squark decays}

We study the effect of the mixing between the 2nd and 3rd generation of squarks on their decays. The branching ratios of the squark decays

$$
\tilde{u}_{1,2} \rightarrow c \tilde{\chi}_{1}^{0} \quad \text { and } \quad \tilde{u}_{1,2} \rightarrow t \tilde{\chi}_{1}^{0}
$$

are calculated by taking into account the following two-body decays:

$$
\tilde{u}_{i} \rightarrow u_{k} \tilde{g}, u_{k} \tilde{\chi}_{n}^{0}, d_{k} \tilde{\chi}_{m}^{+}, \tilde{u}_{j} Z^{0}, \tilde{d}_{j} W^{+}, \tilde{u}_{j} h^{0}
$$

where $u_{k}=(u, c, t)$ and $d_{k}=(d, s, b)$. The decays into the heavier Higgs bosons are kinematically forbidden in our scenarios studied below. The formulae for the widths of the two-body decays in (13) can be found in [13], except for the squark decays into the Higgs boson, for which we take the formulae of $[31,32]$.

We take $\tan \beta, m_{A^{0}}, M_{1}, M_{2}, M_{3}, \mu, M_{Q \alpha \beta}^{2}, M_{U \alpha \beta}^{2}, M_{D \alpha \beta}^{2}, T_{U \alpha \beta}$ and $T_{D \alpha \beta}$ as the basic MSSM parameters at the weak scale and assume them to be real. Here $M_{3}$ is the $\mathrm{SU}(3)$ gaugino mass parameter. The QFV parameters are the squark generation mixing terms $M_{Q \alpha \beta}^{2}, M_{U \alpha \beta}^{2}, M_{D \alpha \beta}^{2}, T_{U \alpha \beta}$ and $T_{D \alpha \beta}$ with $\alpha \neq \beta$. We study a specific scenario which is chosen so that QFV signals at LHC may be maximized and hence can serve as a benchmark scenario for further experimental investigations. As such a scenario, we 


\begin{tabular}{|c|c|c|c|c|c|c|c|c|c|c|c|}
\hline$\tilde{u}_{1}$ & $\tilde{u}_{2}$ & $\tilde{u}_{3}$ & $\tilde{u}_{4}$ & $\tilde{u}_{5}$ & $\tilde{u}_{6}$ & $\tilde{d}_{1}$ & $\tilde{d}_{2}$ & $\tilde{d}_{3}$ & $\tilde{d}_{4}$ & $\tilde{d}_{5}$ & $\tilde{d}_{6}$ \\
\hline \hline 472 & 708 & 819 & 837 & 897 & 918 & 800 & 820 & 830 & 835 & 897 & 922 \\
\hline \hline$\tilde{g}$ & $\tilde{\chi}_{1}^{0}$ & $\tilde{\chi}_{2}^{0}$ & $\tilde{\chi}_{3}^{0}$ & $\tilde{\chi}_{4}^{0}$ & $\tilde{\chi}_{1}^{ \pm}$ & $\tilde{\chi}_{2}^{ \pm}$ & $h^{0}$ & $H^{0}$ & $A^{0}$ & $H^{ \pm}$ \\
\hline \hline 800 & 138 & 261 & 1003 & 1007 & 261 & 1007 & 122 & 800 & 800 & 804 \\
\hline
\end{tabular}

Table 2: Sparticles, Higgs bosons and corresponding masses (in $\mathrm{GeV}$ ) in the scenario of Table $1 . H^{0}$ is the heavier CP-even Higgs boson.

\begin{tabular}{|c||c|c|c|c|c|c|}
\hline $\mid R_{i \alpha}^{\tilde{u} \alpha}$ & $\tilde{u}_{L}$ & $\tilde{c}_{L}$ & $\tilde{t}_{L}$ & $\tilde{u}_{R}$ & $\tilde{c}_{R}$ & $\tilde{t}_{R}$ \\
\hline \hline$\tilde{u}_{1}$ & 0.001 & 0.004 & 0.024 & 0 & 0.715 & 0.699 \\
$\tilde{u}_{2}$ & 0.003 & 0.014 & 0.055 & 0 & 0.699 & 0.713 \\
$\tilde{u}_{3}$ & 0 & 0 & 0 & 1.0 & 0 & 0 \\
$\tilde{u}_{4}$ & 0.128 & 0.584 & 0.800 & 0 & 0.021 & 0.053 \\
$\tilde{u}_{5}$ & 0.181 & 0.781 & 0.598 & 0 & 0.008 & 0.024 \\
$\tilde{u}_{6}$ & 0.975 & 0.221 & 0.005 & 0 & 0 & 0 \\
\hline
\end{tabular}

Table 3: The up-type squark compositions in the flavour eigenstates, i.e. the absolute values of the mixing matrix elements $R_{i \alpha}^{\tilde{u}}$ for the scenario of Table 1 .

take the scenario specified by Table 1, which was studied for QFV gluino decays in [15]. Here we take $M_{1}=(5 / 3) \tan ^{2} \theta_{W} M_{2}$, assuming gaugino mass unification including the gluino mass parameter $M_{3}$. In this scenario one has $\delta_{23}^{u L L}=0.068, \delta_{23}^{u R R}=0.4$ and $\delta_{23}^{u R L}=\delta_{32}^{u R L}=0$ for the QFV parameters. This scenario satisfies the conditions (i)-(v). For the observables in (i) and (ii) we obtain $B(b \rightarrow s \gamma)=3.56 \times 10^{-4}, B\left(b \rightarrow s l^{+} l^{-}\right)=$ $1.59 \times 10^{-6}, B\left(B_{s} \rightarrow \mu^{+} \mu^{-}\right)=4.71 \times 10^{-9}, B\left(B_{u}^{-} \rightarrow \tau^{-} \bar{\nu}_{\tau}\right)=7.85 \times 10^{-5}, \Delta M_{B_{s}}=$ $17.37 \mathrm{ps}^{-1}$ and $\Delta \rho(S U S Y)=1.51 \times 10^{-4}$. The resulting tree-level masses of squarks, neutralinos and charginos are given in Table 2 and the up-type squark compositions in the flavour eigenstates in Table 3.

For the most important decay branching ratios of the two lightest up-type squarks we get $B\left(\tilde{u}_{1} \rightarrow c \tilde{\chi}_{1}^{0}\right)=0.59, B\left(\tilde{u}_{1} \rightarrow t \tilde{\chi}_{1}^{0}\right)=0.39, B\left(\tilde{u}_{2} \rightarrow c \tilde{\chi}_{1}^{0}\right)=0.44, B\left(\tilde{u}_{2} \rightarrow\right.$ $\left.t \tilde{\chi}_{1}^{0}\right)=0.40$. Note that the branching ratios of the decays of a squark into quarks of different generations are very large simultaneously, which could lead to large QFV effects. In our scenario this is a consequence of the facts that both squarks $\tilde{u}_{1,2}$ are mainly 
strong mixtures of $\tilde{c}_{R}$ and $\tilde{t}_{R}$ due to the large $\tilde{c}_{R}-\tilde{t}_{R}$ mixing term $M_{U 23}^{2}\left(=(373 \mathrm{GeV})^{2}\right)$ (see Table 3 ) and that $\tilde{\chi}_{1}^{0}$ is mainly the $U(1)$ gaugino. This also suppresses the couplings of $\tilde{u}_{1,2}$ to $\tilde{\chi}_{2}^{0}$ and $\tilde{\chi}_{1}^{+}$which are mainly $S U(2)$ gauginos. Note that $\tilde{\chi}_{3,4}^{0}$ and $\tilde{\chi}_{2}^{ \pm}$ are very heavy in this scenario.

The main decay branching ratios of the other up-type squarks are as follows: $B\left(\tilde{u}_{3} \rightarrow\right.$ $\left.u \tilde{\chi}_{1}^{0}\right)=0.93, B\left(\tilde{u}_{4} \rightarrow c \tilde{\chi}_{2}^{0}\right)=0.09, B\left(\tilde{u}_{4} \rightarrow t \tilde{\chi}_{2}^{0}\right)=0.21, B\left(\tilde{u}_{4} \rightarrow s \tilde{\chi}_{1}^{+}\right)=0.21, B\left(\tilde{u}_{4} \rightarrow\right.$ $\left.b \tilde{\chi}_{1}^{+}\right)=0.45, B\left(\tilde{u}_{5} \rightarrow c \tilde{\chi}_{2}^{0}\right)=0.19, B\left(\tilde{u}_{5} \rightarrow t \tilde{\chi}_{2}^{0}\right)=0.07, B\left(\tilde{u}_{5} \rightarrow s \tilde{\chi}_{1}^{+}\right)=0.37, B\left(\tilde{u}_{5} \rightarrow\right.$ $\left.b \tilde{\chi}_{1}^{+}\right)=0.17, B\left(\tilde{u}_{5} \rightarrow c \tilde{g}\right)=0.17, B\left(\tilde{u}_{6} \rightarrow u \tilde{\chi}_{2}^{0}\right)=0.22, B\left(\tilde{u}_{6} \rightarrow d \tilde{\chi}_{1}^{+}\right)=0.47$, and $B\left(\tilde{u}_{6} \rightarrow u \tilde{g}\right)=0.28$.

We now study various parameter dependences of the QFV squark decay branching ratios for the reference scenario of Table 1 . In all plots we mark the point corresponding to this scenario by an "x". In Figs.1-3 we show that both $B\left(\tilde{u}_{i} \rightarrow c \tilde{\chi}_{1}^{0}\right)$ and $B\left(\tilde{u}_{i} \rightarrow t \tilde{\chi}_{1}^{0}\right)$ $(\mathrm{i}=1,2)$ can be very large simultaneously in a sizable QFV parameter region satisfying all of the conditions (i)-(v), which can lead to large rates for QFV signal events at LHC as we will see in the next section.

Fig.1 shows the contours of $B\left(\tilde{u}_{1} \rightarrow c \tilde{\chi}_{1}^{0}\right)$ and $B\left(\tilde{u}_{1} \rightarrow t \tilde{\chi}_{1}^{0}\right)$ in the $\left(\Delta M_{U}^{2}, M_{U 23}^{2}\right)$ plane with $\Delta M_{U}^{2} \equiv M_{U 22}^{2}-M_{U 33}^{2}$. The range of $M_{U 23}^{2}$ shown corresponds to the range $\left|\delta_{23}^{u R R}\right|<0.45$ for $\Delta M_{U}^{2}=0$. In the region shown all of the low energy constraints are fulfilled. We see that there are sizable regions where both decay modes are important at the same time. The observed behaviour can be easily understood in the limit where the $\tilde{t}_{L}-\tilde{t}_{R}$ mixing is neglected since in this limit only the mixing between $\tilde{c}_{R}$ and $\tilde{t}_{R}$ is relevant for $\tilde{u}_{1,2}$ and the corresponding effective mixing angle is given by $\tan \left(2 \theta_{\tilde{c}_{R} \tilde{t}_{R}}^{e f f}\right) \equiv 2 M_{U 23}^{2} /\left(\Delta M_{U}^{2}-m_{t}^{2}\right)$. Note that for $\Delta M_{U}^{2}-m_{t}^{2}>0\left[\Delta M_{U}^{2}-m_{t}^{2}<0\right]$, we have $\tilde{u}_{1} \sim \tilde{t}_{R}\left(+\tilde{c}_{R}\right)\left[\tilde{u}_{1} \sim \tilde{c}_{R}\left(+\tilde{t}_{R}\right)\right]$. We also find that the behaviour of $B\left(\tilde{u}_{2} \rightarrow c \tilde{\chi}_{1}^{0}\right)$ and $B\left(\tilde{u}_{2} \rightarrow t \tilde{\chi}_{1}^{0}\right)$ is similar to that of $B\left(\tilde{u}_{1} \rightarrow t \tilde{\chi}_{1}^{0}\right)$ and $B\left(\tilde{u}_{1} \rightarrow c \tilde{\chi}_{1}^{0}\right)$, respectively, which is a consequence of the fact that mainly the mixing between $\tilde{c}_{R}$ and $\tilde{t}_{R}$ is important for the $\tilde{u}_{1,2}$ system.

Fig.2 presents contours of $B\left(\tilde{u}_{2} \rightarrow c \tilde{\chi}_{1}^{0}\right)$ and $B\left(\tilde{u}_{2} \rightarrow t \tilde{\chi}_{1}^{0}\right)$ in the $\delta_{23}^{u L L}-\delta_{23}^{u R R}$ plane where all of the conditions (i)-(v) are satisfied except the $b \rightarrow s \gamma$ constraint which we show by plotting the corresponding $B(b \rightarrow s \gamma)$ contours. All basic parameters other than $M_{Q 23}^{2}$ and $M_{U 23}^{2}$ are fixed as in the scenario of Table 1. For $B\left(\tilde{u}_{1} \rightarrow c \tilde{\chi}_{1}^{0}\right)$ and $B\left(\tilde{u}_{1} \rightarrow t \tilde{\chi}_{1}^{0}\right)$ we have obtained similar contours to Fig.2.(b) and Fig.2.(a), respectively, but they are almost flat. From Fig.2 we find that the possibility of the large QFV 

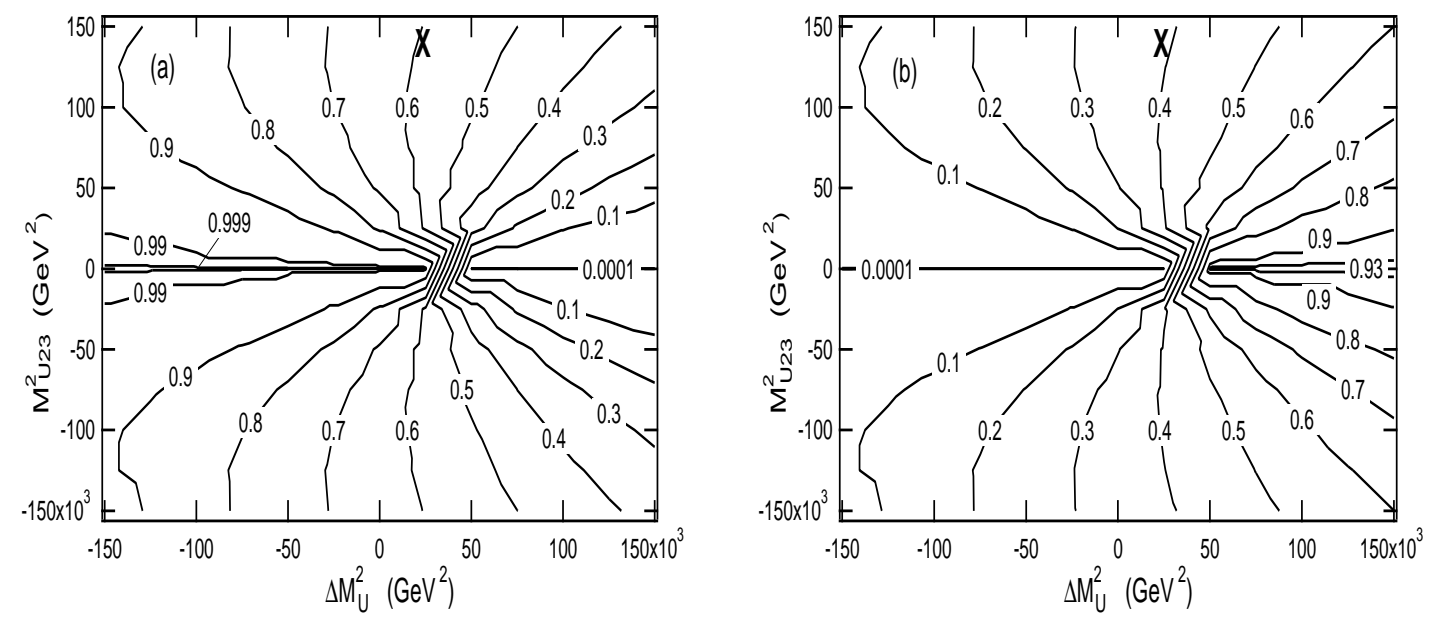

Figure 1: Contours of the QFV decay branching ratios (a) $B\left(\tilde{u}_{1} \rightarrow c \tilde{\chi}_{1}^{0}\right)$ and (b) $B\left(\tilde{u}_{1} \rightarrow\right.$ $\left.t \tilde{\chi}_{1}^{0}\right)$ in the $\left(\Delta M_{U}^{2}, M_{U 23}^{2}\right)$ plane where all of the conditions (i)-(v) are satisfied.

effect cannot be excluded by the $b \rightarrow s \gamma$ constraint even if the experimental error of $B(b \rightarrow s \gamma)$ becomes very small. We see also that $B\left(\tilde{u}_{2} \rightarrow c \tilde{\chi}_{1}^{0}\right)$ and $B\left(\tilde{u}_{2} \rightarrow t \tilde{\chi}_{1}^{0}\right)$ are sensitive [rather insensitive] to $\delta_{23}^{u R R}\left[\delta_{23}^{u L L}\right]$. For large values of $\delta_{23}^{u R R}$ we see that there is a mild dependence on $\delta_{23}^{u L L}$. This is due the fact that for large $\delta_{23}^{u R R}$ the mass squared difference between $\tilde{u}_{2}$ (the heavier of the RR sector, i.e. the $\tilde{c}_{R}-\tilde{t}_{R}$ sector) and $\tilde{u}_{4}$ (the lighter of the LL sector, i.e. the $\tilde{c}_{L}-\tilde{t}_{L}$ sector) becomes small and of the same size as the $\tilde{t}_{L}-\tilde{t}_{R}$ mixing term $\left(=-m_{t} \mu \cot \beta\right.$ (see Eq.(4))) enhancing the mixing between the $\mathrm{RR}$ and LL sectors. For small values of $\delta_{23}^{u R R}$ the RR sector decouples effectively from the LL sector and hence the $\tilde{u}_{2}$ decay branching ratios are almost independent of $\delta_{23}^{u L L}$.

In Fig. 3 we show the $\delta_{23}^{u R L}$ dependences of the $\tilde{u}_{1,2}$ decay branching ratios, where all basic parameters other than $T_{U 32}$ are fixed as in the scenario of Table 1. The observed dependences are a consequence of the enhanced $\tilde{t}_{L}$ component in $\tilde{u}_{1,2}\left(\sim \tilde{c}_{R}+\tilde{t}_{R}\right)$ for increased $\left|\delta_{23}^{u R L}\right|$. The enhanced $\tilde{t}_{L}$ content implies an enhancement of the $b \tilde{\chi}_{1}^{+}\left(\simeq \tilde{W}^{+}\right)$ mode. The enhancement of $B\left(\tilde{u}_{2} \rightarrow \tilde{u}_{1} h^{0}\right)$ for increased $\left|\delta_{23}^{u R L}\right|$ is partly also caused by the enhanced $\tilde{t}_{L}$ component and, more importantly, by the increased coupling of $\tilde{u}_{2} \tilde{u}_{1} h^{0}$ which contains a term proportional to $T_{U 32}$. Note that in such scenarios squark decays could be additional sources of the Higgs boson. The asymmetry with respect to $\delta_{23}^{u R L}=0$ follows from the $\tilde{t}_{L}-\tilde{t}_{R}$ mixing term $\left(=-m_{t} \mu \cot \beta \neq 0\right.$ (see Eq.(4))) which already induces some $\tilde{t}_{L}$ component in $\tilde{u}_{1,2}$ (see Table 3 ). As for the $\delta_{32}^{u R L}$ dependence 

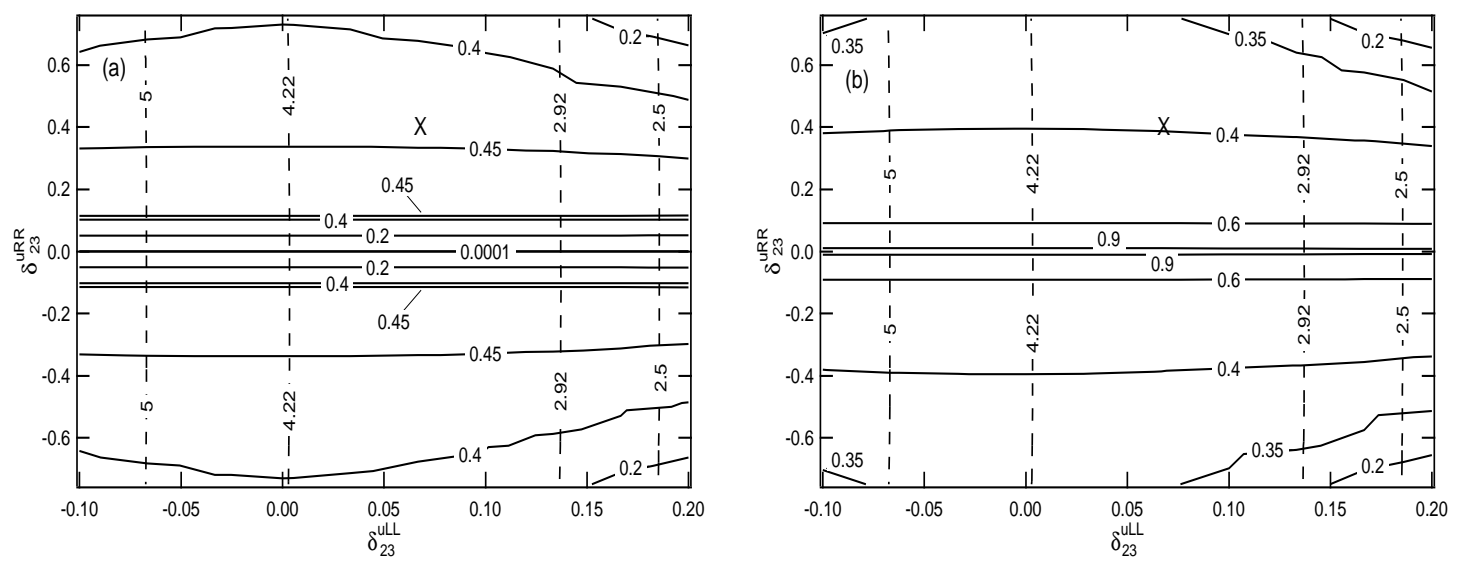

Figure 2: Contours of (a)B( $\left.\tilde{u}_{2} \rightarrow c \tilde{\chi}_{1}^{0}\right)$ and (b) $B\left(\tilde{u}_{2} \rightarrow t \tilde{\chi}_{1}^{0}\right)$ (solid lines) in the $\delta_{23}^{u L L}-$ $\delta_{23}^{u R R}$ plane where all of the conditions (i)-(v) except the $b \rightarrow s \gamma$ constraint are satisfied. Contours of $10^{4} \times B(b \rightarrow s \gamma)$ (dashed lines) are also shown. The condition (i) requires $2.92<10^{4} \times B(b \rightarrow s \gamma)<4.22$.
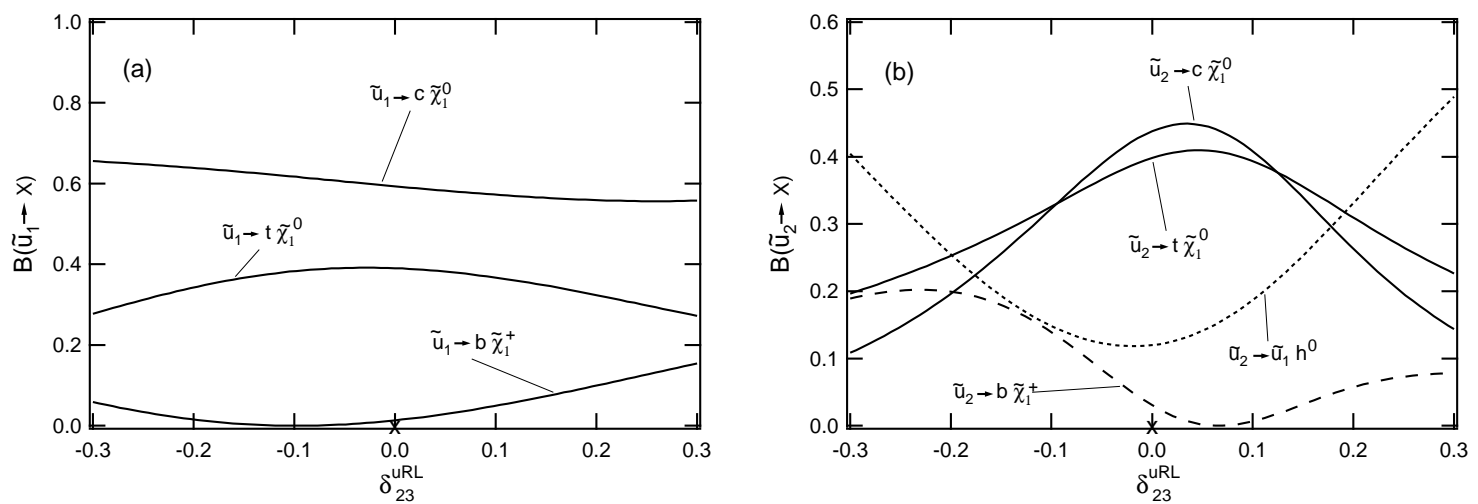

Figure 3: $\delta_{23}^{u R L}$ dependences of the (a) $\tilde{u}_{1}$ and (b) $\tilde{u}_{2}$ decay branching ratios. The shown range of $\delta_{23}^{u R L}$ is the whole range allowed by the conditions (i) to (v) given in the text; note that the range $\left|\delta_{23}^{u R L}\right| \gtrsim 0.3$ is excluded by the condition (v). 
of the $\tilde{u}_{1,2}$ decay branching ratios, we have obtained results similar to those for the $\delta_{23}^{u R L}$ dependence in Fig.3.

\section{$5 \quad$ Impact on collider signatures}

We now study effects of the squark generation mixing on QFV signals at LHC. The large $B\left(\tilde{u}_{i} \rightarrow c \tilde{\chi}_{1}^{0}\right)$ and $B\left(\tilde{u}_{i} \rightarrow t \tilde{\chi}_{1}^{0}\right)(i=1,2)$ may result in a sizable rate for the following QFV signals:

$$
p p \rightarrow \tilde{u}_{1,2} \overline{\tilde{u}}_{1,2} X \rightarrow c \bar{t} \tilde{\chi}_{1}^{0} \tilde{\chi}_{1}^{0} X, t \bar{c} \tilde{\chi}_{1}^{0} \tilde{\chi}_{1}^{0} X
$$

where $\mathrm{X}$ contains only beam-jets and the $\tilde{\chi}_{1}^{0}$ 's give rise to missing transverse energy $E_{T}^{m i s}$. The corresponding cross sections are given by

$$
\begin{aligned}
\sigma_{c t}^{i j} & \equiv \sigma\left(p p \rightarrow \tilde{u}_{i} \overline{\tilde{u}}_{j} X \rightarrow c \bar{t}(t \bar{c}) \tilde{\chi}_{1}^{0} \tilde{\chi}_{1}^{0} X\right) \\
& \equiv \sigma\left(p p \rightarrow \tilde{u}_{i} \overline{\tilde{u}}_{j} X \rightarrow c \bar{t} \tilde{\chi}_{1}^{0} \tilde{\chi}_{1}^{0} X\right)+\sigma\left(p p \rightarrow \tilde{u}_{i} \overline{\tilde{u}}_{j} X \rightarrow t \bar{c} \tilde{\chi}_{1}^{0} \tilde{\chi}_{1}^{0} X\right) \\
& =\sigma\left(p p \rightarrow \tilde{u}_{i} \overline{\tilde{u}}_{j} X\right)\left[B\left(\tilde{u}_{i} \rightarrow c \tilde{\chi}_{1}^{0}\right) \cdot B\left(\overline{\tilde{u}}_{j} \rightarrow \bar{t}_{\tilde{\chi}}^{0}\right)+B\left(\tilde{u}_{i} \rightarrow t \tilde{\chi}_{1}^{0}\right) \cdot B\left(\overline{\tilde{u}}_{j} \rightarrow \bar{c} \tilde{\chi}_{1}^{0}\right)\right] .
\end{aligned}
$$

We calculate the relevant squark-squark and squark-antisquark pair production cross sections at leading order using the WHIZARD/O'MEGA packages [33, 34] where we have implemented the model described in Section 2 with squark generation mixing in its most general form. We use the CTEQ6L global parton density fit [35] for the parton distribution functions and take $Q=m_{\tilde{u}_{i}}+m_{\tilde{u}_{j}}$ for the factorization scale, where $\tilde{u}_{i}$ and $\tilde{u}_{j}$ are the squark pair produced. The QCD coupling $\alpha_{s}(Q)$ is also evaluated (at the two-loop level) at this scale Q. We have cross-checked our implementation of QFV by comparing with the results obtained using the public packages FeynArts [36] and FormCalc [37].

Defining QFC production cross sections as

$$
\begin{aligned}
\sigma_{q \bar{q}}^{i j} & \equiv \sigma\left(p p \rightarrow \tilde{u}_{i} \overline{\tilde{u}}_{j} X \rightarrow q \bar{q} \tilde{\chi}_{1}^{0} \tilde{\chi}_{1}^{0} X\right) \\
& =\sigma\left(p p \rightarrow \tilde{u}_{i} \overline{\tilde{u}}_{j} X\right) \cdot B\left(\tilde{u}_{i} \rightarrow q \tilde{\chi}_{1}^{0}\right) \cdot B\left(\overline{\tilde{u}}_{j} \rightarrow \bar{q} \tilde{\chi}_{1}^{0}\right) \quad(q=c, t),
\end{aligned}
$$

we obtain the following cross sections at the center-of-mass energy $E_{c m}=14 \mathrm{TeV}[7$ $\mathrm{TeV}]$ in the scenario of Table 1: $\sigma_{c t}^{11}=172.8[11.8] \mathrm{fb}, \sigma_{c t}^{22}=11.5[0.41] \mathrm{fb}, \sigma_{c \bar{c}}^{11}=131.4$ 
$[9.0] \mathrm{fb}, \sigma_{c \bar{c}}^{22}=6.3[0.23] \mathrm{fb}, \sigma_{t \bar{t}}^{11}=56.8[3.89] \mathrm{fb}, \sigma_{t \bar{t}}^{22}=5.2[0.19] \mathrm{fb}$. The expected number of the $c \bar{t} / t \bar{c}$ production events of Eq. (14) is $\mathcal{L} \cdot \sum_{i, j=1,2} \sigma_{c t}^{i j} \simeq 18400$ [10] events for an integrated luminosity of $\mathcal{L}=100 \mathrm{fb}^{-1}\left[1 \mathrm{fb}^{-1}\right]$ at LHC with $E_{c m}=14 \mathrm{TeV}$ $[7 \mathrm{TeV}]$.

The main contribution to $\sigma\left(p p \rightarrow \tilde{u}_{i} \overline{\tilde{u}}_{i} X\right)(i=1,2)$ comes from the subprocess $g g \rightarrow$ $\tilde{u}_{i} \overline{\tilde{u}}_{i}$. The gluon- $\tilde{u}_{i}-\tilde{u}_{j}$ coupling vanishes for $i \neq j$ due to the color SU(3) symmetry. Therefore, $\sigma\left(p p \rightarrow \tilde{u}_{i} \overline{\tilde{u}}_{j} X\right)$ and hence $\sigma_{c t}^{i j}$, $\sigma_{c \bar{c}}^{i j}$ and $\sigma_{t \bar{t}}^{i j}$ are very small for $i \neq j$, e.g. $\mathrm{O}(0.01) \mathrm{fb}\left[\mathrm{O}\left(10^{-4}\right) \mathrm{fb}\right]$ for $(i, j)=(1,2)$ at $E_{c m}=14 \mathrm{TeV}[7 \mathrm{TeV}]$. We have found that the production cross sections of the quark pair $(c \bar{t}, t \bar{c}, c \bar{c}, t \bar{t})$ plus two $\tilde{\chi}_{1}^{0}$ 's and $n \nu$ 's $(n=0,2,4, \ldots)$ via production of the heavier up-type squarks $\tilde{u}_{i}(i \geq 3)$ are very small in this scenario.

In Fig. 4 we show the $\delta_{23}^{u R R}$ dependences of the QFV production cross sections $\sigma_{c t}^{i i}$ $(i=1,2)$ at $E_{c m}=7$ and $14 \mathrm{TeV}$, where all basic parameters other than $M_{U 23}^{2}$ are fixed as in the scenario of Table 1. The QFV cross sections at $14 \mathrm{TeV}$ are about an order of magnitude larger than those at $7 \mathrm{TeV}$. We see that the QFV cross sections quickly increase with increase of the QFV parameter $\left|\delta_{23}^{u R R}\right|$ around $\delta_{23}^{u R R}=0$ and that they can be quite sizable in a wide allowed range of $\delta_{23}^{u R R}$. The mass of $\tilde{u}_{1}\left(\tilde{u}_{2}\right)$ decreases (increases) with increase of $\left|\delta_{23}^{u R R}\right|$. This leads to the increase of $\sigma_{c t}^{11}$ and the decrease of $\sigma_{c t}^{22}$ with increase of $\left|\delta_{23}^{u R R}\right| . \sigma_{c t}^{11}$ vanishes for $\left|\delta_{23}^{u R R}\right| \gtrsim 0.76$, where the decay $\tilde{u}_{1} \rightarrow t \tilde{\chi}_{1}^{0}$ is kinematically forbidden. We have $\tilde{u}_{2}=\tilde{u}_{R}$ for $\left|\delta_{23}^{u R R}\right| \gtrsim 0.9$, which explains the enhancement of $\sigma\left(p p \rightarrow \tilde{u}_{2} \overline{\tilde{u}}_{2} X\right)$ and the vanishing of $\sigma_{c t}^{22}$ for $\left|\delta_{23}^{u R R}\right| \gtrsim 0.9$. Note that in case $\tilde{u}_{2}=\tilde{u}_{R}$, the subprocess $u \bar{u} \rightarrow \tilde{u}_{2}\left(=\tilde{u}_{R}\right) \overline{\tilde{u}}_{2}\left(=\overline{\tilde{u}}_{R}\right)$ via t-channel gluino exchange also can contribute to $\sigma\left(p p \rightarrow \tilde{u}_{2} \overline{\tilde{u}}_{2} X\right)$.

We have also studied the $\delta_{32}^{u R L}$ dependence of the QFV production cross sections $\sigma_{c t}^{i i}(i=1,2)$ at $E_{c m}=7 \mathrm{TeV}$ and $14 \mathrm{TeV}$, where all basic parameters other than $T_{U 23}$ are fixed as in the scenario of Table 1 . We find that the QFV cross sections are rather insensitive to the QFV parameter $\delta_{32}^{u R L}$ and that they can be large in a wide allowed range $\left|\delta_{32}^{u R L}\right| \lesssim 0.3: \sigma_{c t}^{11} \sim 170[10] \mathrm{fb}, \sigma_{c t}^{22} \sim 10[0.4] \mathrm{fb}$ at $E_{c m}=14 \mathrm{TeV}[7$ $\mathrm{TeV}]$. The masses of $\tilde{u}_{1,2}$ decrease (and hence the cross sections $\sigma^{i i}(i=1,2)$ increase) and the branching ratios $B\left(\tilde{u}_{1,2} \rightarrow c / t \tilde{\chi}_{1}^{0}\right)$ tend to decrease with increase of $\left|\delta_{32}^{u R L}\right|$. This implies that the QFV cross sections are rather insensitive to $\delta_{32}^{u R L}$. As for the $\delta_{23}^{u R L}$ dependence of $\sigma_{c t}^{i i}(i=1,2)$ we have obtained similar results to those for the $\delta_{32}^{u R L}$ dependence. 

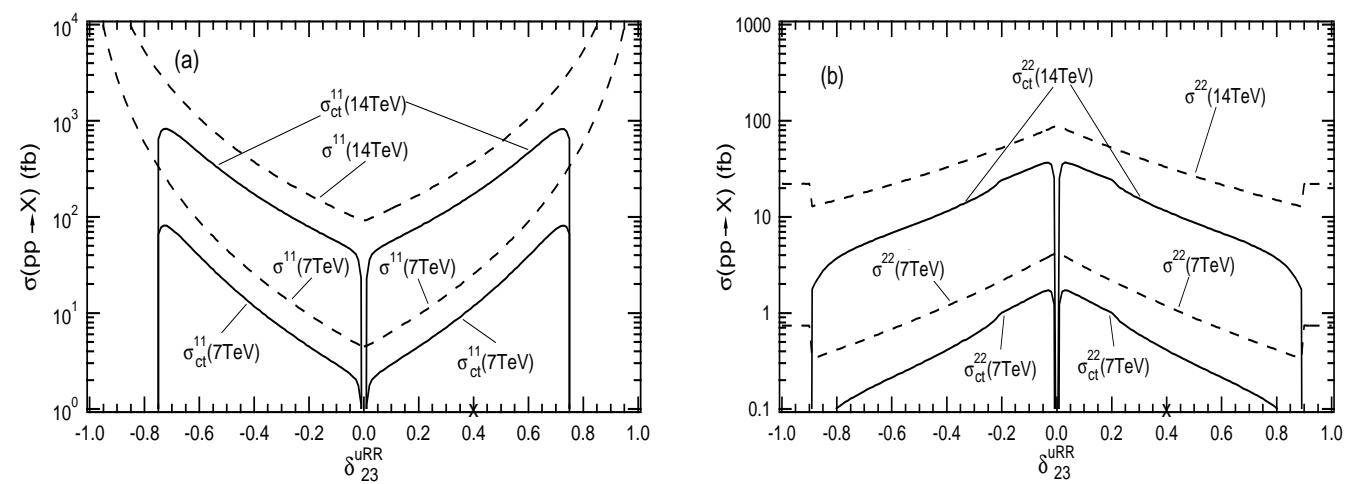

Figure 4: $\delta_{23}^{u R R}$ dependences of (a) $\sigma^{11} \equiv \sigma\left(p p \rightarrow \tilde{u}_{1} \overline{\tilde{u}}_{1} X\right), \sigma_{c t}^{11}$ and (b) $\sigma^{22} \equiv \sigma(p p \rightarrow$ $\left.\tilde{u}_{2} \overline{\tilde{u}}_{2} X\right), \sigma_{c t}^{22}$ at $E_{c m}=7$ and $14 \mathrm{TeV}$. The point "x" of $\delta_{23}^{u R R}=0.4$ corresponds to our reference scenario of Table 1 . The shown range of $\delta_{23}^{u R R}$ is the whole range allowed by the conditions (i) to $(\mathrm{v})$ given in the text; note that the range $\left|\delta_{23}^{u R R}\right| \gtrsim 1.0$ is excluded by the condition $m_{\tilde{u}_{1}}>m_{\tilde{\chi}_{1}^{0}}$ in (iii).

The large $\tilde{c}_{R}-\tilde{t}_{R}$ mixing could also give rise to the following QFV production cross sections:

$$
\begin{aligned}
\sigma_{t t}^{i j} & \equiv \sigma\left(p p \rightarrow \tilde{u}_{i} \tilde{u}_{j} X \rightarrow t t \tilde{\chi}_{1}^{0} \tilde{\chi}_{1}^{0} X\right) \\
& =\sigma\left(p p \rightarrow \tilde{u}_{i} \tilde{u}_{j} X\right) \cdot B\left(\tilde{u}_{i} \rightarrow t \tilde{\chi}_{1}^{0}\right) \cdot B\left(\tilde{u}_{j} \rightarrow t \tilde{\chi}_{1}^{0}\right)(i, j=1,2)
\end{aligned}
$$

where $\mathrm{X}$ contains only beam-jets. Here the $\tilde{u}_{i} \tilde{u}_{j}$ pair (with $\tilde{u}_{i, j} \sim \tilde{c}_{R}+\tilde{t}_{R}$ in the scenario under consideration) is produced mainly via a t-channel gluino exchange subprocess $c c \rightarrow \tilde{u}_{i} \tilde{u}_{j}$ with $\mathrm{c}$ being the charm-quark in the beam proton. Note that the signal event "top-quark + top-quark $+E_{T}^{m i s}+$ beam-jets" can practically not be produced in the MSSM with QFC (nor in the SM). It turns out however that in the scenario of Table 1 the corresponding cross section $\sigma_{t t} \equiv \sigma_{t t}^{11}+\sigma_{t t}^{12}$ is at most $\mathrm{O}(0.1) \mathrm{fb}$ at $E_{c m}=14$ $\mathrm{TeV}$ and hence that it might be relevant for a very high luminosity [38]. Therefore this QFV process will not be discussed further.

In addition, we study QFV in production and decays of squarks at LHC for a QFV scenario based on the mSUGRA scenario SPS1a' [39] which has served as input for several experimental studies. The high energy inputs at the GUT scale $M_{G U T}=$ $2.47 \times 10^{16} \mathrm{GeV}$ in the scenario SPS1a' are taken as $m_{0}=70 \mathrm{GeV}, m_{1 / 2}=250 \mathrm{GeV}$, $A_{0}=-300 \mathrm{GeV}$ and $\mu>0$ together with $\tan \beta\left(m_{Z}\right)=10$. Here $m_{0}, m_{1 / 2}$ and $A_{0}$ are the common scalar mass, gaugino mass and trilinear coupling at the GUT scale, 


\begin{tabular}{|c||c|c|c|}
\hline$M_{Q \alpha \beta}^{2}$ & $\beta=1$ & $\beta=2$ & $\beta=3$ \\
\hline \hline$\alpha=1$ & $(526)^{2}$ & 0 & 0 \\
\hline$\alpha=2$ & 0 & $(526)^{2}$ & 0 \\
\hline$\alpha=3$ & 0 & 0 & $(471)^{2}$ \\
\hline
\end{tabular}

\begin{tabular}{|c|c|c|c|c|c|}
\hline$M_{1}$ & $M_{2}$ & $M_{3}$ & $\mu$ & $\tan \beta$ & $m_{A^{0}}$ \\
\hline \hline 103 & 193 & 572 & 398 & 10 & 373 \\
\hline \hline$T_{U 11}$ & $T_{U 22}$ & $T_{U 33}$ & $T_{D 11}$ & $T_{D 22}$ & $T_{D 33}$ \\
\hline \hline-0.007 & -2.68 & -488 & -0.19 & -3.26 & -128 \\
\hline
\end{tabular}

\begin{tabular}{|c||c|c|c|}
\hline$M_{D \alpha \beta}^{2}$ & $\beta=1$ & $\beta=2$ & $\beta=3$ \\
\hline \hline$\alpha=1$ & $(505)^{2}$ & 0 & 0 \\
\hline$\alpha=2$ & 0 & $(505)^{2}$ & 0 \\
\hline$\alpha=3$ & 0 & 0 & $(501)^{2}$ \\
\hline
\end{tabular}

\begin{tabular}{|c||c|c|c|}
\hline$M_{U \alpha \beta}^{2}$ & $\beta=1$ & $\beta=2$ & $\beta=3$ \\
\hline \hline$\alpha=1$ & $(508)^{2}$ & 0 & 0 \\
\hline$\alpha=2$ & 0 & $(508)^{2}$ & $(280)^{2}$ \\
\hline$\alpha=3$ & 0 & $(280)^{2}$ & $(387)^{2}$ \\
\hline
\end{tabular}

Table 4: The MSSM parameters at the scale $\mathrm{Q}=1 \mathrm{TeV}$ in the $\mathrm{QFV}$ scenario based on the SPS1a' scenario. $T_{U \alpha \beta}$ and $T_{D \alpha \beta}$ are set to zero for $\alpha \neq \beta$. All mass parameters are given in $\mathrm{GeV}$. Note that $M_{U 23}^{2}=0$ in the original SPS1a' scenario.

respectively. We use SPheno v3.0 [30] to obtain the resulting MSSM parameters at the scale $\mathrm{Q}=1 \mathrm{TeV}$ according to the SPA convention [39]. At this scale, we add the QFV parameters (i.e. the squark generation mixing parameters) and vary them around zero (i.e. around the MFV scenario). An example set of the MSSM parameters thus obtained is given in Table 4 and the resulting mass spectrum and the up-type squark compositions in the flavour eigenstates in Tables 5 and 6 , respectively. In this scenario one has $\delta_{23}^{u L L}=0, \delta_{23}^{u R R}=0.4$ and $\delta_{23}^{u R L}=\delta_{32}^{u R L}=0$ for the QFV parameters at the scale $\mathrm{Q}=1 \mathrm{TeV}$. Note that the resulting squark and gluino masses are smaller than those in the scenario of Table 1 . We have checked that all of the constraints in Section 3 are fulfilled in this scenario. For the important squark decay branching ratios we obtain $B\left(\tilde{u}_{1} \rightarrow c \tilde{\chi}_{1}^{0}\right)=0.100, B\left(\tilde{u}_{1} \rightarrow t \tilde{\chi}_{1}^{0}\right)=0.230, B\left(\tilde{u}_{2} \rightarrow c \tilde{\chi}_{1}^{0}\right)=0.146, B\left(\tilde{u}_{2} \rightarrow\right.$ $\left.t \tilde{\chi}_{1}^{0}\right)=0.004$. In this scenario the squark mass eigenstate $\tilde{u}_{1}\left(\tilde{u}_{2}\right)$ is dominated by a strong mixture of the flavour eigenstates $\tilde{t}_{R}, \tilde{t}_{L}$ and $\tilde{c}_{R}\left(\tilde{t}_{L}\right.$ and $\left.\tilde{c}_{R}\right)$ and $\tilde{\chi}_{1}^{0}$ is nearly the $\mathrm{U}(1)$ gaugino $\tilde{B}^{0}$ which couples to the right up-type squarks sizably. This explains the sizable branching ratios of $B\left(\tilde{u}_{1} \rightarrow c \tilde{\chi}_{1}^{0}\right), B\left(\tilde{u}_{1} \rightarrow t \tilde{\chi}_{1}^{0}\right)$ and $B\left(\tilde{u}_{2} \rightarrow c \tilde{\chi}_{1}^{0}\right)$ and the very small $B\left(\tilde{u}_{2} \rightarrow t \tilde{\chi}_{1}^{0}\right)$. In this scenario we obtain the following cross sections at the center-of-mass energy $E_{c m}=14 \mathrm{TeV}[7 \mathrm{TeV}]: \sigma_{c t}^{11}=119.7[11.8] \mathrm{fb}, \sigma_{c t}^{22}=0.197$ [0.01] fb. Note that the QFV decay branching ratios $B\left(\tilde{u}_{1,2} \rightarrow c / t \tilde{\chi}_{1}^{0}\right)$ are significantly smaller than those in the scenario of Table 1 , but that the QFV production cross section $\sigma_{c t}^{11}$ is nevertheless large due to the lighter squarks in this scenario based on SPS1a'. 


\begin{tabular}{|c|c|c|c|c|c|c|c|c|c|c|c|c|c|c|}
\hline$\tilde{u}_{1}$ & $\tilde{u}_{2}$ & $\tilde{u}_{3}$ & & $\tilde{u}_{4}$ & $\tilde{u}_{5}$ & $\tilde{u}_{6}$ & $\tilde{d}_{1}$ & $\tilde{d}_{2}$ & c & $l_{3}$ & $\tilde{d}_{4}$ & $d$ & & $\tilde{d}_{6}$ \\
\hline 332 & 541 & 54 & & 665 & 565 & 612 & 506 & 54 & & 47 & 547 & 57 & & 571 \\
\hline & $\tilde{g}$ & $\tilde{\chi}_{1}^{0}$ & $\tilde{\chi}_{2}^{0}$ & $\tilde{\chi}_{3}^{0}$ & $\tilde{\chi}_{4}^{0}$ & $\tilde{\chi}$ & & & $h^{0}$ & $H$ & & & 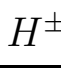 & \\
\hline & 08 & 98 & 184 & 402 & 415 & 18 & & & 112 & 42 & & 126 & 43 & \\
\hline
\end{tabular}

Table 5: Sparticles, Higgs bosons and corresponding physical masses (in $\mathrm{GeV}$ ) in the scenario of Table 4.

\begin{tabular}{|c||c|c|c|c|c|c|}
\hline$\left|R_{i \alpha}^{\tilde{u}}\right|$ & $\tilde{u}_{L}$ & $\tilde{c}_{L}$ & $\tilde{t}_{L}$ & $\tilde{u}_{R}$ & $\tilde{c}_{R}$ & $\tilde{t}_{R}$ \\
\hline \hline$\tilde{u}_{1}$ & 0.010 & 0.032 & 0.457 & 0 & 0.369 & 0.809 \\
$\tilde{u}_{2}$ & 0.014 & 0.015 & 0.691 & 0 & 0.720 & 0.062 \\
$\tilde{u}_{3}$ & 0 & 0 & 0 & 1.0 & 0 & 0 \\
$\tilde{u}_{4}$ & 0.896 & 0.444 & 0.011 & 0 & 0.003 & 0.001 \\
$\tilde{u}_{5}$ & 0.443 & 0.893 & 0.036 & 0 & 0.062 & 0.008 \\
$\tilde{u}_{6}$ & 0.021 & 0.058 & 0.559 & 0 & 0.585 & 0.585 \\
\hline
\end{tabular}

Table 6: The up-type squark compositions in the flavour eigenstates, i.e. the absolute values of the mixing matrix elements $R_{i \alpha}^{\tilde{u}}$, at the scale $\mathrm{Q}=1 \mathrm{TeV}$ for the scenario of Table 4.

In Fig.5 we show the $\delta_{23}^{u R R}$ dependence of the QFV production cross section $\sigma_{c t}^{11}$ at $E_{c m}=7 \mathrm{TeV}$ and $14 \mathrm{TeV}$, where all basic parameters other than $M_{U 23}^{2}(Q=1 \mathrm{TeV})$ are fixed as in the scenario of Table $4 . \sigma_{c t}^{22}$ is very small due to the very small $B\left(\tilde{u}_{2} \rightarrow t \tilde{\chi}_{1}^{0}\right)$. We see that the QFV cross section increases with increase of the QFV parameter $\left|\delta_{23}^{u R R}\right|$ and that it can be quite sizable in a wide allowed range of $\delta_{23}^{u R R}$. The mass of $\tilde{u}_{1}$ decreases and $B\left(\tilde{u}_{1} \rightarrow c \tilde{\chi}_{1}^{0}\right) \cdot B\left(\tilde{u}_{1} \rightarrow t \tilde{\chi}_{1}^{0}\right)$ increases with increase of $\left|\delta_{23}^{u R R}\right|$. This leads to the increase of $\sigma_{c t}^{11}$ with increase of $\left|\delta_{23}^{u R R}\right| . \sigma_{c t}^{11}$ vanishes for $\left|\delta_{23}^{u R R}\right| \gtrsim 0.62$, where the decay $\tilde{u}_{1} \rightarrow t \tilde{\chi}_{1}^{0}$ is kinematically forbidden.

Finally, we briefly discuss the detectability of the QFV production process $p p \rightarrow$ $\tilde{u}_{i} \overline{\tilde{u}}_{i} X \rightarrow c \bar{t}(t \bar{c}) \tilde{\chi}_{1}^{0} \tilde{\chi}_{1}^{0} X(i=1,2)$ at LHC. The signature is '(anti)top-quark + charm-jet $+E_{T}^{m i s}+X^{\prime}$, where $\mathrm{X}$ contains beam-jets only. Therefore, identifying the top-quarks in the final states is mandatory. This should be possible by using the hadronic decays of the top-quark. Charm-tagging would also be very useful. There is another QFV signal 


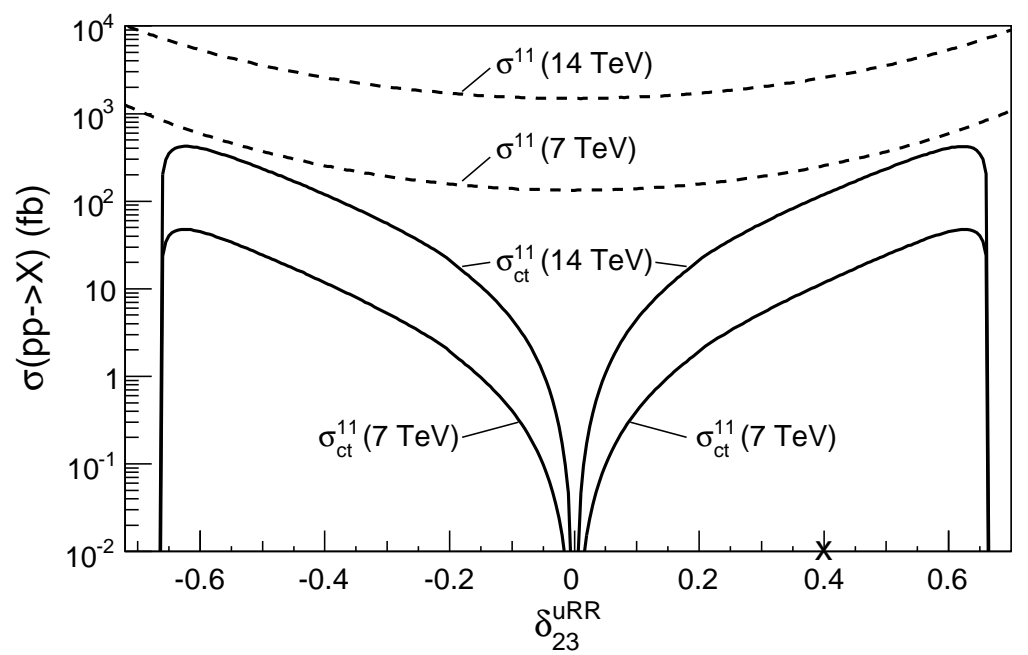

Figure 5: $\delta_{23}^{u R R}$ dependences of $\sigma^{11} \equiv \sigma\left(p p \rightarrow \tilde{u}_{1} \overline{\tilde{u}}_{1} X\right)$ and $\sigma_{c t}^{11}$ at $E_{c m}=7 \mathrm{TeV}$ and 14 TeV. The point " $\mathrm{x}$ " of $\delta_{23}^{u R R}=0.4$ corresponds to the QFV scenario of Table 4. The shown range of $\delta_{23}^{u R R}$ is allowed by the conditions (i) to (v) given in the text.

process leading to the same final states, i.e. gluino production and its QFV decay [15] $p p \rightarrow \tilde{g} \tilde{\chi}_{1}^{0} X \rightarrow c \bar{t}(t \bar{c}) \tilde{\chi}_{1}^{0} \tilde{\chi}_{1}^{0} X$. This cross section is, however, about a factor of 20-30 smaller than that of the QFV process via squark pair production in the scenarios studied here. This suppression is mainly due to the electroweak interactions involved. The QFV production process $p p \rightarrow c \bar{t}(t \bar{c}) X$ via SUSY-QCD one-loop diagrams [40] also yields the signature '(anti)top-quark + charm-jet $+\mathrm{X}$ '. The size of the cross section of this process is of the same order as that of our QFV process Eq.(14). However, the missing- $E_{T}$ would be much smaller than that in our signal process Eq. (14). If charm-tagging is not possible, one should search for the process $p p \rightarrow \tilde{u}_{i} \overline{\tilde{u}}_{i} X \rightarrow$ $q \bar{t}(t \bar{q}) \tilde{\chi}_{1}^{0} \tilde{\chi}_{1}^{0} X(q \neq t, b)$, i.e. for the signature '(anti)top-quark + jet $+E_{T}^{m i s}+X^{\prime}$. Main backgrounds are single top-quark productions in the SM. The most important one is due to tW production where the W-boson decays into a tau-lepton which then decays hadronically: $p p \rightarrow t W X \rightarrow t \tau \nu X \rightarrow t \tau$-jet $\nu \bar{\nu} X$. The cross section for the tW production $\sigma\left(p p \rightarrow t W^{-} X\right)+\sigma\left(p p \rightarrow \bar{t} W^{+} X\right)$ is about $66 \mathrm{pb}$ for $E_{c m}=14 \mathrm{TeV}$ [41]. It turns out that the $\mathrm{W}$-boson is mainly produced in the central region (see e.g. [42]). To reduce this background one can use the fact that a charm-quark jet has usually a much higher particle-multiplicity than the $\tau$-jet. By requiring that at least four hadrons are contained in the jet, this background cross section can be reduced to 
about $12 \mathrm{fb}$ for $E_{c m}=14 \mathrm{TeV}$ without significant loss of our QFV signal events. One can expect that it is much smaller than $12 \mathrm{fb}$ for $E_{c m}=7 \mathrm{TeV}$. In the case that one considers only hadronic decays of the top-quark, one can require in addition that the invariant mass of each jet is larger than the tau-lepton mass, which should reduce this background further again without significant loss of our signal events.

The second important background is single top-quark production due to t-channel W-boson exchange in the SM. Relevant for us is the reaction $p p \rightarrow t(\bar{t}) q(\bar{q}) Z^{0} X \rightarrow$ $t(\bar{t}) q(\bar{q}) \nu \bar{\nu} X$, where the main contribution is due to W-boson exchange in the t-channel (quite similar to $p p \rightarrow t q X$, for which a thorough treatment is given in [17]). Using the WHIZARD/O'MEGA package $[33,34]$ we have calculated the corresponding cross sections and obtained at $E_{c m}=14 \mathrm{TeV}[7 \mathrm{TeV}]$ :

$\sigma\left(p p \rightarrow t q Z^{0} X \rightarrow t q \nu \bar{\nu} X\right)=97.4[17.5] \mathrm{fb}$,

$\sigma\left(p p \rightarrow t \bar{q} Z^{0} X \rightarrow t \bar{q} \nu \bar{\nu} X\right)=15.9[1.89] \mathrm{fb}$

$\sigma\left(p p \rightarrow \bar{t} q Z^{0} X \rightarrow \bar{t} q \nu \bar{\nu} X\right)=46.0[7.1] \mathrm{fb}$,

$\sigma\left(p p \rightarrow \bar{t} \bar{q} Z^{0} X \rightarrow \bar{t} \bar{q} \nu \bar{\nu} X\right)=13.2[1.6] \mathrm{fb}$,

where the cross sections summed over $q=u, d, c, s$ are shown. Comparing these to Figs. 4 and 5 we see that for $\left|\delta_{23}^{u R R}\right| \gtrsim 0.45$ our signal cross section is larger than the sum of these background cross sections. For $\left|\delta_{23}^{u R R}\right| \lesssim 0.45$ a suitable $E_{T}^{m i s}$ cut will reduce this background relatively to our signal because the $E_{T}^{m i s}$ due to $\nu \bar{\nu}$ from the $Z^{0}$ decay will on the average be smaller than that due to the two neutralinos.

The cross section of single top-quark production via s-channel $\mathrm{W}$-boson exchange is much smaller than that of our QFV process. We obtain for $E_{c m}=14 \mathrm{TeV}[7 \mathrm{TeV}]$ :

$\sigma\left(p p \rightarrow " W^{+} " Z^{0} X \rightarrow t \bar{b} \nu \bar{\nu} X\right)=1.42[0.45] \mathrm{fb}$,

$\sigma\left(p p \rightarrow " W^{-"} Z^{0} X \rightarrow \bar{t} b \nu \bar{\nu} X\right)=0.73[0.18] \mathrm{fb}$.

There could be another background from the QFC top-quark pair production processes (a) $p p \rightarrow \tilde{u}_{i} \overline{\tilde{u}}_{i} X \rightarrow t \bar{t} \tilde{\chi}_{1}^{0} \tilde{\chi}_{1}^{0} X$ and (b) $p p \rightarrow t \bar{t} Z^{0} X \rightarrow t \bar{t} \nu \bar{\nu} X$, where one of the Wbosons from the top-quarks decays leptonically with the charged lepton being missed. However, the probability of such W-boson decay would be very small. Moreover, these top-quark pair production cross sections are not so large compared to our QFV cross sections. For the process (a), for example, we see that $\sigma_{t \bar{t}}^{i i}<\sigma_{c t}^{i i}(i=1,2)$ in the scenario studied as shown just after Eq. (16). For (b) we obtain at $E_{c m}=14 \mathrm{TeV}[7$ $\mathrm{TeV}]: \sigma\left(p p \rightarrow t \bar{t} Z^{0} X \rightarrow t \bar{t} \nu \bar{\nu} X\right)=97.6[13.8] \mathrm{fb}$.

Of course, a detailed Monte Carlo study including detector effects is required for a proper assessment of the detectability of the proposed QFV signal. However, this 
is beyond the scope of this paper and will be presented in a forthcoming publication [43].

\section{Conclusion}

To conclude, we have studied the effects of squark mixing of the second and third generation, especially $\tilde{c}_{L / R}-\tilde{t}_{L / R}$ mixing, on squark production and decays at LHC in the MSSM. We have shown that the effect can be very large in a significant region of the QFV parameters despite the very strong constraints on QFV from experimental data on $\mathrm{B}$ mesons. The QFV squark decay branching ratios $\mathrm{B}\left(\tilde{u}_{i} \rightarrow c \tilde{\chi}_{1}^{0}\right)$ and $\mathrm{B}\left(\tilde{u}_{i} \rightarrow\right.$ $\left.t \tilde{\chi}_{1}^{0}\right)(i=1,2)$ can be very large (up to $\left.\sim 50 \%\right)$ simultaneously. This can result in QFV signal events ' $p p \rightarrow c \bar{t}(t \bar{c})+E_{T}^{m i s}+$ beam-jets' with a significant rate at LHC. The observation of these remarkable signatures would provide a powerful test of supersymmetric QFV at LHC. Therefore, in the squark search one should take into account the possibility of significant contributions from QFV squark decays. Moreover, one should also include the QFV squark parameters (i.e. the squark generation mixing parameters) in the determination of the basic SUSY parameters at LHC.

\section{Acknowledgments}

This work is supported by the "Fonds zur Förderung der wissenschaftlichen Forschung (FWF)" of Austria, project No. P18959-N16. The authors acknowledge support from EU under the MRTN-CT-2006-035505 network program. B. H. and W. P. are supported by the DFG, project No. PO 1337/1-1. The work of B. H. is supported in part by the Landes-Exzellenzinitiative Hamburg. W. P. is supported by the Alexander von Humboldt Foundation and the Spanish grant FPA2008-00319/FPA.

\section{References}

[1] A. J. Buras, P. Gambino, M. Gorbahn, S. Jager and L. Silvestrini, Phys. Lett. B 500 (2001) 161 [arXiv:hep-ph/0007085]. 
[2] G. D'Ambrosio, G. F. Giudice, G. Isidori and A. Strumia, Nucl. Phys. B 645 (2002) 155 [arXiv:hep-ph/0207036].

[3] A. L. Kagan, G. Perez, T. Volansky and J. Zupan, Phys. Rev. D 80 (2009) 076002 [arXiv:0903.1794 [hep-ph]].

[4] G. Colangelo, E. Nikolidakis and C. Smith, Eur. Phys. J. C 59 (2009) 75 [arXiv:0807.0801 [hep-ph]].

[5] G. D. Kribs, E. Poppitz and N. Weiner, Phys. Rev. D 78 (2008) 055010 [arXiv:0712.2039 [hep-ph]].

[6] N. Arkani-Hamed, J. L. Feng, L. J. Hall and H. C. Cheng, Nucl. Phys. B 505 (1997) 3 [arXiv:hep-ph/9704205].

[7] G. Hiller, Y. Hochberg and Y. Nir, JHEP 0903 (2009) 115 [arXiv:0812.0511 [hep$\mathrm{ph}]]$.

[8] see e.g. J. J. Liu et al., Nucl. Phys. B 705 (2005) 3 [arXiv:hep-ph/0404099]; S. Bejar, J. Guasch and J. Sola, JHEP 0510 (2005) 113 [arXiv:hep-ph/0508043]; G. Eilam, M. Frank and I. Turan, Phys. Rev. D 74 (2006) 035012 [arXiv:hepph/0601253]; J. J. Cao et al., Phys. Rev. D 75 (2007) 075021 [arXiv:hepph/0702264]; D. Lopez-Val, J. Guasch and J. Sola, JHEP 0712 (2007) 054 [arXiv:0710.0587 [hep-ph]]; S. Bejar et al., Phys. Lett. B 668 (2008) 364 [arXiv:0805.0973 [hep-ph]].

[9] see e.g. C. S. Li, R. J. Oakes and J. M. Yang, Phys. Rev. D 49 (1994) 293 [Erratum-ibid. D 56 (1997) 3156]; G. Couture, C. Hamzaoui and H. Konig, Phys. Rev. D 52 (1995) 1713 [arXiv:hep-ph/9410230]; J. M. Yang and C. S. Li, Phys. Rev. D 49 (1994) 3412 [Erratum-ibid. D 51 (1995) 3974]; J. L. Lopez, D. V. Nanopoulos and R. Rangarajan, Phys. Rev. D 56 (1997) 3100 [arXiv:hepph/9702350]; J. Guasch and J. Sola, Nucl. Phys. B 562 (1999) 3 [arXiv:hepph/9906268]; J. L. Diaz-Cruz, H. J. He and C. P. Yuan, Phys. Lett. B 530 (2002) 179 [arXiv:hep-ph/0103178]; J. Cao et al., Phys. Rev. D 74 (2006) 031701 [arXiv:hep-ph/0604163].

[10] A. M. Curiel, M. J. Herrero and D. Temes, Phys. Rev. D 67 (2003) 075008 [arXiv:hep-ph/0210335]; D. A. Demir, Phys. Lett. B 571 (2003) 193 [arXiv:hep- 
ph/0303249]; A. M. Curiel et al., Phys. Rev. D 69 (2004) 075009 [arXiv:hepph/0312135]; S. Bejar et al., JHEP 0408 (2004) 018 [arXiv:hep-ph/0402188]; T. Hahn et al., arXiv:hep-ph/0512315.

[11] K. i. Hikasa and M. Kobayashi, Phys. Rev. D 36 (1987) 724; T. Han et al., Phys. Rev. D 70 (2004) 055001 [arXiv:hep-ph/0312129]; E. Lunghi, W. Porod and O. Vives, Phys. Rev. D 74 (2006) 075003 [arXiv:hep-ph/0605177]; G. Hiller and Y. Nir, JHEP 0803 (2008) 046 [arXiv:0802.0916 [hep-ph]].

[12] W. Porod, JHEP 0205 (2002) 030 [arXiv:hep-ph/0202259].

[13] G. Bozzi et al., Nucl. Phys. B 787 (2007) 1 [arXiv:0704.1826 [hep-ph]].

[14] B. Fuks, B. Herrmann and M. Klasen, Nucl. Phys. B 810 (2009) 266 [arXiv:0808.1104 [hep-ph]]; G. D. Kribs, A. Martin and T. S. Roy, arXiv:0901.4105 [hep-ph].

[15] A. Bartl, K. Hidaka, K. Hohenwarter-Sodek, T. Kernreiter, W. Majerotto and W. Porod, Phys. Lett. B 679 (2009) 260 [arXiv:0905.0132 [hep-ph]].

[16] T. Hurth and W. Porod, JHEP 0908 (2009) 087 [arXiv:0904.4574 [hep-ph]].

[17] T. Plehn, M. Rauch and M. Spannowsky, Phys. Rev. D 80 (2009) 114027 [arXiv:0906.1803 [hep-ph]].

[18] B. Allanach et al., Comput. Phys. Commun. 180 (2009) 8 [arXiv:0801.0045 [hep$\mathrm{ph}]$.

[19] F. Gabbiani, E. Gabrielli, A. Masiero and L. Silvestrini, Nucl. Phys. B 477 (1996) 321 [arXiv:hep-ph/9604387].

[20] T. Iijima, plenary talk at The XXIV International Symposium on Lepton Photon Interactions at High Energies (Lepton Photon 09), Hamburg, 17-22 August 2009.

[21] M. Misiak et al., Phys. Rev. Lett. 98 (2007) 022002 [arXiv:hep-ph/0609232].

[22] M. Iwasaki et al. [Belle Collaboration], Phys. Rev. D72 (2005) 092005 [arXiv:hepex/0503044]; B. Aubert et al. [BABAR Collaboration], Phys. Rev. Lett. 93 (2004) 081802 [arXiv:hep-ex/0404006]; see also E. Lunghi, W. Porod and O. Vives, Phys. Rev. D74 (2006) 075003 [arXiv:hep-ph/0605177]. 
[23] R. Sangro, plenary talk at the 17th International Conference on Supersymmetry and the Unification of Fundamental Interactions (SUSY09), Boston, 5-10 June 2009, AIP Conf. Proc. 1200 (2010) 22.

[24] A. Abulencia et al. [CDF Collaboration], Phys. Rev. Lett. 97 (2006) 242003.

[25] M. Carena et al., Phys. Rev. D74 (2006) 015009 [arXiv:hep-ph/0603106]; see also P. Ball and R. Fleischer, Eur. Phys. J. C48 (2006) 413 [arXiv:hep-ph/0604249].

[26] G. Altarelli, R. Barbieri, F. Caravaglios, Int. J. Mod. Phys. A13 (1998) 1031.

[27] G. Sguazzoni, Proceedings of the 31st International Conference on High Energy Physics, Amsterdam, The Netherlands, 25 - 31 July 2002, Eds. S. Bentvelson, P. de Jong, J. Koch, E. Laenen, p. 709 [arXiv:hep-ex/0210022]; P. Lutz, the same Proceedings, p. 735; S. Schael et al., ALEPH Collaboration and DELPHI Collaboration and L3 Collaboration and OPAL Collaborations and LEP Working Group for Higgs Boson Searches, Eur. Phys. J. C 47 (2006) 547.

[28] K. Peters, talk at The 34th International Conference on High Energy Physics (ICHEP 2008), Philadelphia, 30 July to 5 August 2008; M. D'Onofrio, talk at the same conference.

[29] J. A. Casas and S. Dimopoulos, Phys. Lett. B 387 (1996) 107 [arXiv:hep$\mathrm{ph} / 9606237]$.

[30] The code SPheno v3.0 can be obtained from http://theorie.physik.uni-wuerzburg.de/ porod/SPheno.html; W. Porod, Comput. Phys. Commun. 153 (2003) 275 [arXiv:hep-ph/0301101].

[31] A. Bartl, S. Hesselbach, K. Hidaka, T. Kernreiter and W. Porod, Phys. Rev. D 70 (2004) 035003 [arXiv:hep-ph/0311338].

[32] M. Bruhnke, B. Herrmann and W. Porod, arXiv:1007.2100 [hep-ph].

[33] W. Kilian, T. Ohl and J. Reuter, arXiv:0708.4233 [hep-ph].

[34] M. Moretti, T. Ohl and J. Reuter, arXiv:hep-ph/0102195.

[35] J. Pumplin et al., JHEP 0207 (2002) 012, [arXiv:hep-ph/0201195]. 
[36] T. Hahn, Comput. Phys. Commun. 140 (2001) 418 [arXiv:hep-ph/0012260]; T. Hahn and C. Schappacher, Comput. Phys. Commun. 143 (2002) 54 [arXiv:hep$\mathrm{ph} / 0105349]$.

[37] T. Hahn and M. Perez-Victoria, Comput. Phys. Commun. 118 (1999) 153 [arXiv:hep-ph/9807565].

[38] M. Nessi, plenary talk at The XXIV International Symposium on Lepton Photon Interactions at High Energies (Lepton Photon 09), Hamburg, 17-22 August 2009.

[39] J.A. Aguilar-Saavedra et al., Eur. Phys. J. C46 (2006) 43 [arXiv:hep-ph/0511344].

[40] J. Guasch, W. Hollik, S. Penaranda and J. Sola, Nucl. Phys. Proc. Suppl. 157 (2006) 152 [arXiv:hep-ph/0601218].

[41] M. Beneke et al., arXiv:hep-ph/0003033.

[42] E. Re, arXiv:1009.2450 [hep-ph].

[43] B. Herrmann et al., in preparation. 